Classification

Physics Abstracts

$02.50-\mathrm{r}-07.80+\mathrm{x}-85.40 \mathrm{Ci}$

\title{
3D statistics from TEM observations of TPFG EEPROM memory cells
}

Paolo Mengucci $\left({ }^{1}\right)$, Daniele Rinaldi $\left({ }^{1}\right)$, Stefano Santini $\left({ }^{2}\right)$ and Massimo Vanzi $\left({ }^{3}\right)$

( $\left.{ }^{1}\right)$ Dipartimento di Scienze dei Materiali e della Terra, Università di Ancona, via Brecce Bianche, 60131 Ancona, Italy

$\left({ }^{2}\right)$ Istituto di Fisica e Bioingegneria, Università di Urbino, via S. Chiara, 61029 Urbino, Italy

( $\left.{ }^{3}\right)$ Telettra S.p.A., Q\& R, via Capo di Lucca 31, 40126 Bologna, Italy

(Received June 04, 1990; accepted October 08, 1990)

Résumé. - Des dispositifs commerciaux EEPROM fabriqués selon la technologie TPFG (textured polysilicon floating gate) ont été observés en coupe par microscopie électronique à transmission. Comme prévu, de nombreuses pointes sont visibles sur la surface du silicium polycristallin où l'émission tunnel d'électrons se produit lors du cycle ECRITURE/EFFACEMENT. On propose un modele statistique reliant les distributions $2 \mathrm{D}$ observées de pointes avec la distribution 3D réelle.

\begin{abstract}
Commercial EEPROM devices based on Textured Polysilicon Floating Gate (TPFG) technology were observed by TEM in cross-sectional view. At the polysilicon surfaces where electron tunneling takes place during the WRITE/ERASE cycle many tips where detected, as expected. A statistical model correlating the observed $2 \mathrm{D}$ tip distribution with the relevant $3 \mathrm{D}$ distribution is proposed.
\end{abstract}

\section{Introduction.}

The widespread application of EEPROM (Electrically Erasable Programmable Read Only Memories) in high technology equipment strongly calls for reliability evaluations on that kind of advanced devices. The critical point lies on the very essential mechanism of the write/erase (WE) process, based on electron tunneling across a $\mathrm{Si} / \mathrm{SiO}_{2}$ potential barrier [1-4], which has been shown prone to cause charge trapping [5-8] at the interface, resulting in an exponential decay of the tunneling probability in time, and then reducing the WE efficiency up to an actual failure state [9-10].

The study of the WE mechanism and of its degradation is complicated in the so called TPFG technology by the technological realization of EEPROM cells, based on local field enhancement at the Si surface by texturing a polysilicon film before its ultimate oxidation [11-16]. The ideal flat surface dealt with in the classical Fowler-Nordheim (FN) framework for electron tunneling is here substituted by an highly irregular distribution of conducting tips, of various sizes and shapes, separated from a smoother conducting surface by the insulating $\mathrm{SiO}_{2}$ layer. 
From the theoretical point of view almost nothing survives of the basic assumptions underlying the FN approach: the flat surface, indeed, is essential for the validity of any unidimensional model leading to the evaluation of image force (the key for the Schottky effect on the potential barrier height), for the simple description of the electric field across the tunneling region, and for the solution of the Schroedinger equation in the WKB approximation, leading to the tunneling coefficient.

Even charge trapping is hardly described in such a situation.

Many attempts have been made to simplify some parts of the general problem, by means of particular geometrical models for the tips, numerical evaluations of the electric field, statistical interpretation of experimental results $[9,17-19]$. In any case, when figures have to be extracted for a specific device technology, the actual distribution of the injecting tips should be obtained.

This can be done by means of Electron Microscopy, in the Scanning (SEM) or Transmission (TEM) mode, provided the specimen are observed in cross-sectional view: It is the only way, indeed, to observe not only the relative tip heights but, what is essential, their distance from the upper conducting surface. Anyway, a cross-sectional view just gives the intersection of a 3D distribution with a plane, and the observed distribution of shapes and heights cannot be taken as a direct measurement of the original 3D situation.

There is a significant indication that the exact shape of the tips is not critical, which is, on the contrary, the case for their maximum height [18].

As a preliminary step for a more general study of the EEPROM degradation process, a method is proposed, now, to evaluate the 3D distribution of tip heights starting from experimental TEM observations of cross-sectional specimens of commercial EEPROM devices: The statistical link from the observed and the required distributions will be explained for the simple assumption of conical shapes.

\section{The specimen and its preparation for TEM.}

2.1 EEPROM PRINCIPLE AND TECHNOLOGY. - The wide success of EEPROMs lies on their nature of Read Only Memories (ROMs) which are both Programmable (PROMs) and Erasable (EPROMs) by electric means. This eliminates the most troubling characteristic of EPROMs, that is the requirement of UV irradiation of the memory cell area for erasing a programmed device.

The technological approach for such a capability calls for electron tunneling across the potential barrier of a dielectric layer separating two polycrystalline conductors [13-16]. Referring to figure 1, the simplified scheme of a single memory cell is represented by an insulated polysilicon gate, which controls the switching of a MOS transistor. Outside of the MOS channel, the gate electrode is sandwiched between two other conductors, separated by a very thin $\mathrm{SiO}_{2}$ dielectric layer. By applying a suitable voltage between those two conductors, it is possible to inject electrons into the floating gate by tunneling (WRITE function), or to extract those electrons in order to return to the initial uncharged condition (ERASE function). When a sufficient amount of charge is stored into the gate, it produces in the MOS channel a field able to switch on or off the transistor. The state of that transistor can then be read many times as in usual EPROM technology: The required voltages for reading that logical state are low enough to avoid any further tunneling to or from the gate, which acts now as a perfectly insulated conductor.

Anyway [16], a very critical process control is required to produce reliable flat thin oxides in the tunneling region. To bypass that problem a rough surface was proposed as the injecting electrode, in order to take advantage of local field enhancement at the vertex of any conducting tip. A field high enough to produce significant tunneling from the tip is reached for an oxide thickness many times larger than in the flat surface case. 


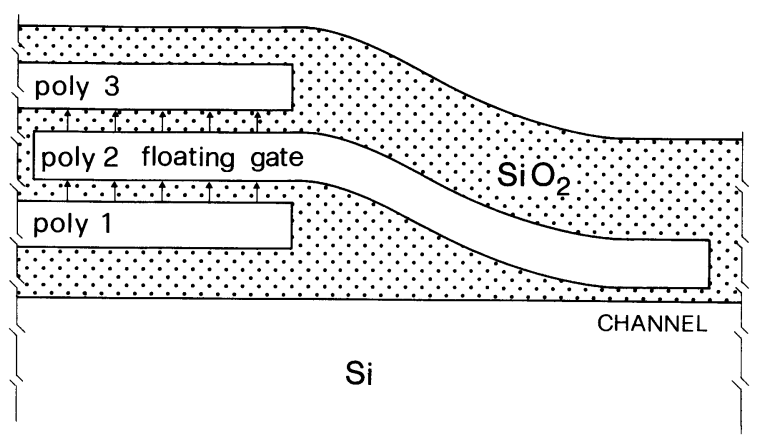

Fig. 1. - Simplified scheme of a single EEPROM cell. Arrows indicate the electron flow direction during tunneling.

The technological process able to produce those structures is the steam oxidation of textured polysilicon: it involves Low Pressure Chemical Vapor Deposition (LPCVD) of a polysilicon layer (typical, $\mathrm{SiH}_{4}$ at $620^{\circ} \mathrm{C}$ ) onto a thermally oxidized single crystal silicon substrate, phosporous doping by $\mathrm{POCl}_{3}$ diffusion (leading to a resistivity of about $10 \mathrm{ohm}$.square in the specific case of the observed devices), phosporous glass removal and a steam oxidation. Depending on details of specific technologies, the last oxide can be kept in place or removed before a new dry oxidation and oxide annealing. In any case, a small grain size is obtained for that polycrystalline microstructure and the preferential oxidation of grain boundaries generates a very rough interface between the conducting and the oxidized part of the polysilicon layer.

That process is applied for the lower conductor of figure 1 and for the floating gate. The upper conductor is left as a standard polysilicon layer. The rough surfaces are so localized at the top of the lower conductor and at the top of the floating gate: This produces a preferential direction for the electron tunneling, from the tips to the upper flat surface, which improves the efficiency of the WRITE or ERASE function.

The typical size of the polysilicon grains in the textured film is about $100 \mathrm{~nm}$, which is comparable with the mean thickness of the interpoly oxide.

The actual geometries of a group of memory cells are reported in the plan view of figure 2, where a MOS channel is indicated and one of the injection areas is crossed by the two lines $\mathrm{A}$ and $\mathrm{B}$.

2.2 TEM AND ITS REQUIREMENTS. - Because of the reliability concern, it is the distribution of the tips to be investigated first. The overall dimensions of the injecting areas call for deep submicrometrical resolution, which means for Transmission Electron Microscopy.

The major limitation of that technique lies on specimen preparation: The ultimate thickness of the area to be investigated must not exceed some tens of nanometers, and a very careful preparation is required in order to reach that figure without introducing misleading artefacts into the thinned specimens.

In order to make feasible a specimen preparation technique proposed by Garulli et al. [20], some commercial chips of TPFG EEPROM were first thermally dismounted from their ceramic package, and their wire bondings were scratched away: in that way no bump exceeded the height of the passivated surface of each chip, as required in the quoted reference. 


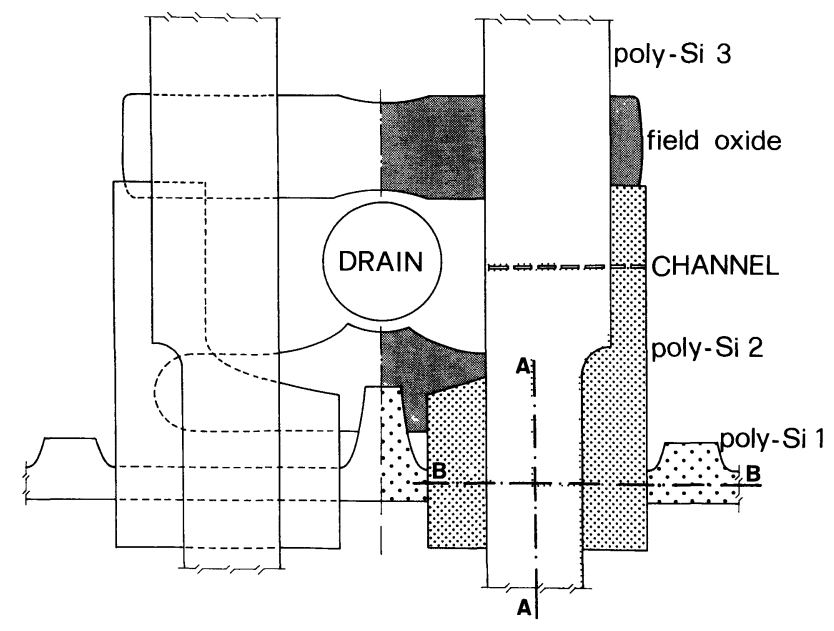

Fig. 2. - Planar scheme of a group of four EEPROM memory cells. The lines AA and BB cross the injection area.
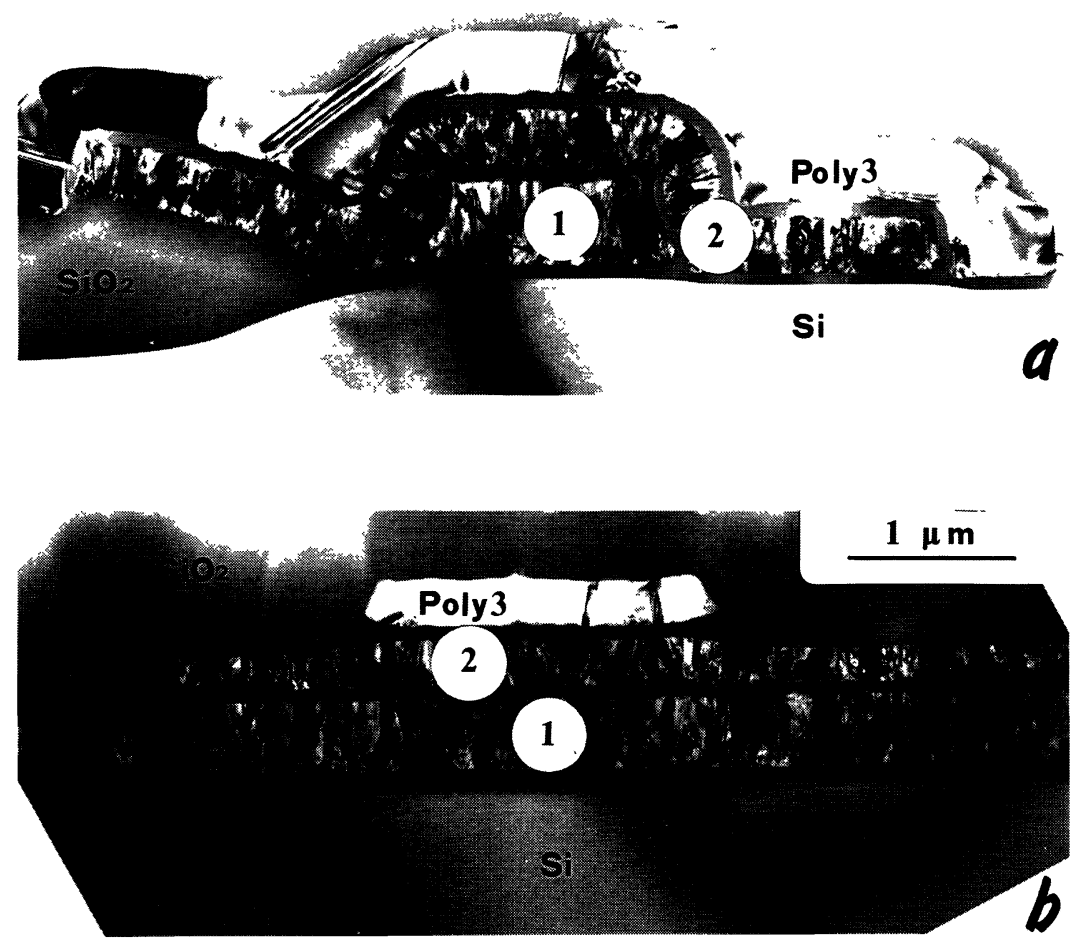

Fig. 3. - TEM cross-sectional images of the injection area of a cell:

a) section along the AA line of figure 2;

b) section along the $\mathrm{BB}$ line. 
The following steps (sandwiching, cutting, grinding and lapping, and final ion milling) were performed according to the standards of that technique.

Two perpendicular directions were chosen to cut the sandwiches, in order to expose both sides of the injection area. Figures $3 a$ and $3 b$ show the overlap of the three polysilicon layers in the two projections: Parallel to the AA line of figure 2 in (a) and to the horizontal edge $\mathrm{BB}$ of the same in (b). The enlarged view of figure 4 displays the corrugated surface of the upper side of the polysilicon gate, and sections of some tips are evident.

From a set of those pictures some statistics can be made about heights and spacing and shapes of the sections of the tips, but not directly of the tips originating those sections. Figure 5 illustrates how an arbitrary distribution of conical tips would produce a cross sectional view (dashed) which clearly underestimates the distribution of the maximum heigths.

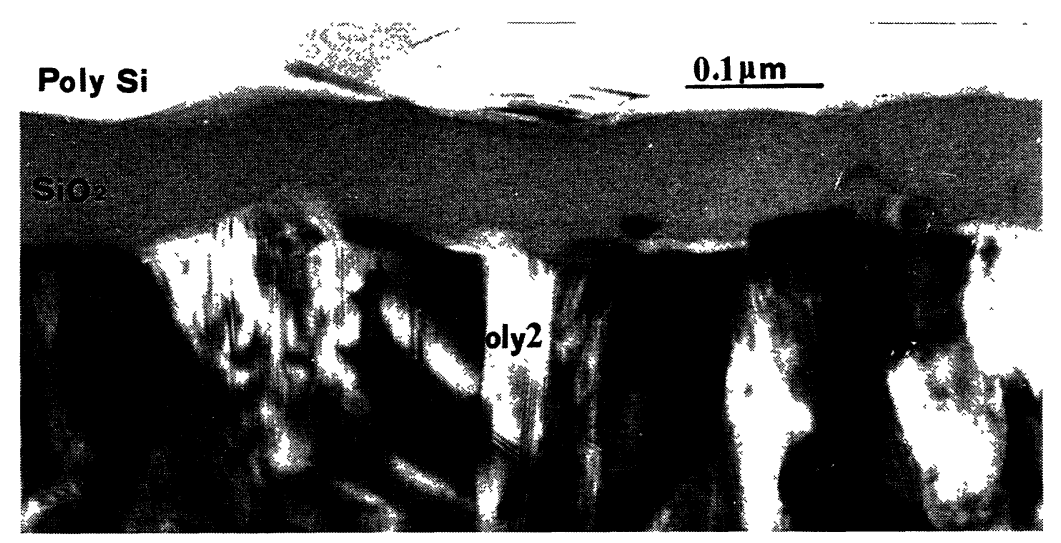

Fig. 4. - Enlarged view of the corrugated polysilicon surface, where electron tunneling is enhanced because of the high density of field lines around the tips.

Moreover, the specimen thickness in the thinnest region, where the pictures were taken, is of the order of some tens of nanometers, which is slightly less than the mean lateral size of the observed asperities. This means that more than one of the smaller tips may stand along the same observation direction, which could modify statistical evaluations.

Some simplified geometrical model of tip distribution is required to properly read the TEM images.

\section{A model for reading TEM cross sectional images.}

3.1 THE MODEL. - As a starting simplification, each tip was described as a cone of fixed $\pi / 2$ aperture and $z_{0}$ height (Fig. 6). With those assumptions the radius of the base is $r_{0}=z_{0}$. Depending on the distance $y_{0}$ of the center of the base circle from the cutting line (intended along the $x$ axis of a $x, y, z$ referential where $y=0$ is the cutting plane), the cone could intersect the cutting plane (for $y_{0}<z_{0}$ ) or not (for $y_{0}>z_{0}$ ). In the first case a hyperbolic shape is drawn on that plane, of maximum height $z_{\mathrm{m}}=z_{0}-y_{0}$, which is the measurable parameter of the TEM observation. Figure 7 reports an experimental shape (a) and its possible generation by a cone (b). A discussion about the simplified assumptions of this model is given at the end of its presentation. 

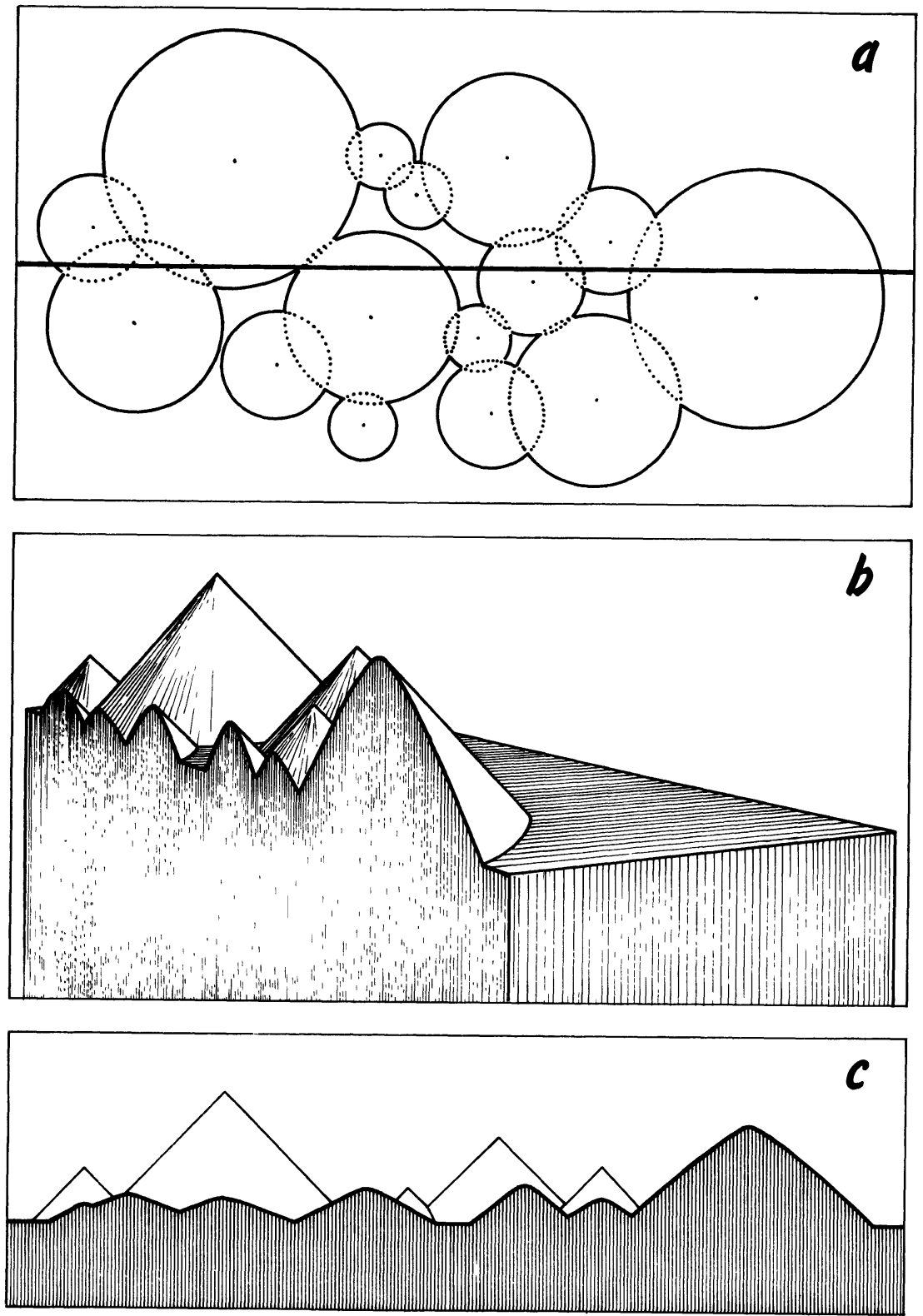

Fig. 5. - An ideal distribution of conical tips and a possible cross sectional view: a) base; b) perspective view; c) planar projection perpendicular to the section plane. The observed maxima in c) are always lower than the actual tip heights.

A distribution of many of such cones will be defined by the position of the centers of their circular bases on the $x, y$ plane and by their respective heights. We assumed that cones are allowed to overlap, and that an uniform surface density $\sigma$ can describe the distribution of the base centers. A linear density $f(z)$ gives account of the height distribution, according to a normalization 


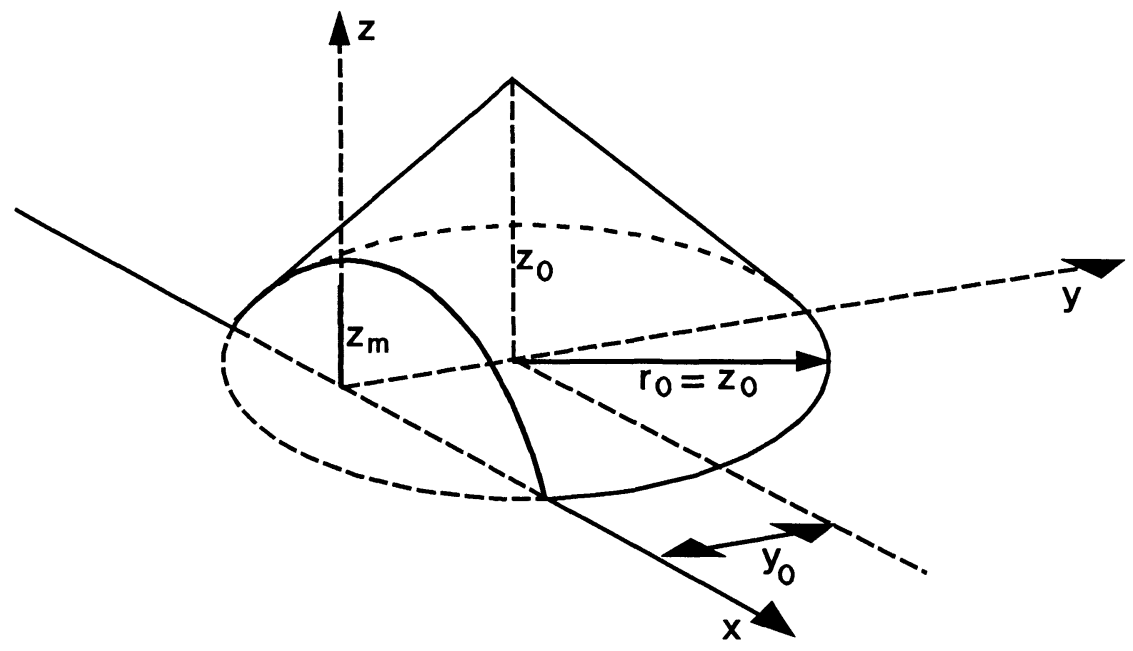

Fig. 6. - The simplified conical model representing a single polysilicon tip, and its cross-section.

condition:

$$
\frac{1}{z_{\mathrm{M}}} \int_{0}^{z_{\mathrm{M}}} f\left(z_{0}\right) \mathrm{d} z_{0}=1
$$

where $z_{M}$ is the maximum value of $z_{0}$. The limited domain $0<z_{0}<z_{\mathrm{M}}$ is required in order to describe the actual situation, where $z_{\mathrm{M}}$ can be seen as the distance of the conducting surface to the base plane of the injecting tips.

From those definitions, the function

$$
\rho\left(x, y, z_{0}\right)=\rho\left(z_{0}\right)=\sigma f\left(z_{0}\right)
$$

will be seen as the density of cones of height $z_{0}$ per unit area. It follows that in a stripe of length $L$ and width $\mathrm{d} y$, located at a distance $y$ from the $y=0$ plane, the linear density in $z_{0}$ will be

$$
\sigma \operatorname{Ld} y f\left(z_{0}\right)
$$

and the number of cones whose vertex lies between $z_{0}$ and $z_{0}+\mathrm{d} z_{0}$ is:

$$
\mathrm{d} n\left(y, z_{0}\right)=\sigma L \mathrm{~d} y f\left(z_{0}\right) \frac{\mathrm{d} z_{0}}{z_{\mathrm{M}}}
$$

All those cones will intersect the $y=0$ plane if $y<z_{0}$. In that case, the height $z_{\mathrm{m}}$ of their intersection will be:

$$
z_{\mathrm{m}}=z_{0}-y
$$

and the limits $0<z_{\mathrm{m}}<z_{\mathrm{M}}$ will hold. This means that the given number $d n\left(y, z_{0}\right)$ will give 

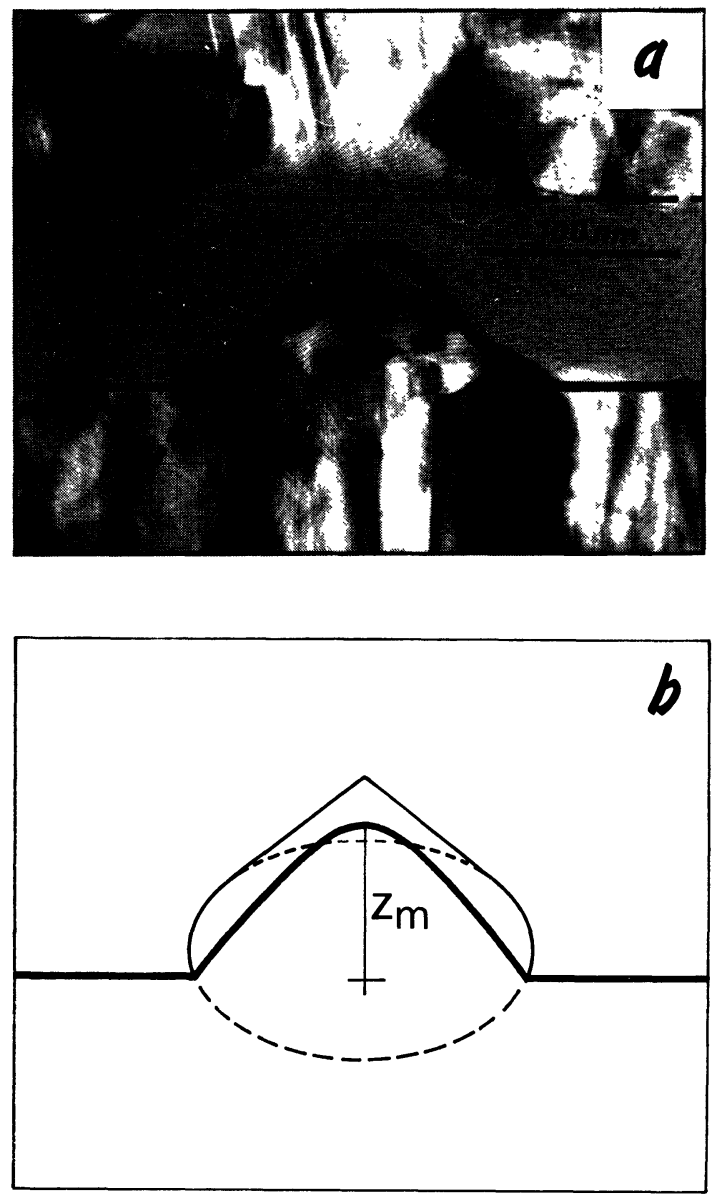

Fig. 7. - An experimental shape (a) is approximated by a parabolic trend. Its generation by a sectioned cone is shown in $b$.

an identical number $\mathrm{d} n\left(y, z_{\mathrm{m}}\right)$ of sections on that plane, provided those limiting conditions are satisfied:

$$
\mathrm{d} n\left(y, z_{\mathrm{m}}\right)= \begin{cases}\mathrm{d} n\left(y, z_{0}\right) & \text { when } 0<z_{0}-y<z_{\mathrm{M}} \\ 0 & \text { otherwise }\end{cases}
$$

Taking into account the relationship between $z_{0}$ and $z_{\mathrm{m}}$, this can be rewritten, at the first order, as:

$$
\mathrm{d} n\left(y, z_{\mathrm{m}}\right)= \begin{cases}\sigma L f\left(y+z_{\mathrm{m}}\right) \mathrm{d} y \frac{\mathrm{d}\left(y+z_{\mathrm{m}}\right)}{z_{\mathrm{M}}} \sim \sigma L f\left(y+z_{\mathrm{m}}\right) \mathrm{d} y \mathrm{~d} z_{\mathrm{m}} & \text { when }\left\{\begin{array}{l}
0<z_{\mathrm{m}}<z_{\mathrm{M}} \\
y+z_{\mathrm{m}}<z_{\mathrm{M}}
\end{array}\right. \\
0 & \text { otherwise }\end{cases}
$$


The same contribution will be given by a symmetrical stripe located at $-y$, and any other couple of symmetrical stripes will give sections of height $z_{\mathrm{m}}$ when relation (1) is satisfied. This indicates that the total number $\mathrm{d} n\left(z_{\mathrm{m}}\right)$ of sections of height between $z_{\mathrm{m}}$ and $z_{\mathrm{m}}+\mathrm{d} z_{\mathrm{m}}$ is given by

$$
\mathrm{d} n\left(z_{\mathrm{m}}\right)=2 \int_{y>0} \mathrm{~d} n\left(y, z_{\mathrm{m}}\right)=\frac{\mathrm{d} z_{\mathrm{m}}}{z_{\mathrm{M}}} 2 \sigma L \int_{0}^{z^{\mathrm{M}^{-} z_{\mathrm{m}}}} f\left(y+z_{\mathrm{m}}\right) \mathrm{d} y=\frac{\mathrm{d} z_{\mathrm{m}}}{z_{\mathrm{M}}} 2 \sigma L \int_{z_{\mathrm{m}}}^{{ }^{\mathrm{M}}} f(p) \mathrm{d} p
$$

The sum over the possible values of $z_{\mathrm{m}}$ gives the total number $N$ of sectioned cones along the distance $L$ :

$$
N=\frac{1}{z_{\mathrm{M}}} \int_{0}^{z_{\mathrm{M}}} \mathrm{d} z_{\mathrm{m}} 2 \sigma L \int_{z_{\mathrm{m}}}^{z_{\mathrm{M}}} f(p) \mathrm{d} p
$$

and the function

$$
\gamma\left(z_{\mathrm{m}}\right)=\frac{1}{N} \frac{\mathrm{d} n\left(z_{\mathrm{m}}\right)}{\mathrm{d} z_{\mathrm{m}}}=\frac{\int_{z \mathbf{m}}^{z \mathrm{M}} f(p) \mathrm{d} p}{\int_{0}^{z} \mathrm{M} \mathrm{d} q \int_{q}^{z \mathrm{M}} f(p) \mathrm{d} p}
$$

can be seen as the linear density of sections of height between $z_{\mathrm{m}}$ and $z_{\mathrm{m}}+\mathrm{d} z_{\mathrm{m}}$ which can be detected on the section plane per unit length.

The above result should indicate that the distribution of the heights over the $x, y$ plane is merely proportional to the derivative of the measured distribution of the heights on the cross sectional plane.

A singificant complication arises when overlap is taken into account: the section of a given cone can be partially or completely hidden by one or more neighbouring cones, and its contribution to the statistical model applied to the measured results should be lost.

Let the center of the base of the given cone be located in $x=0, y=y_{0}$, and let $z_{0}$ be its height. The maximum of its intersection is then defined:

$$
\left\{\begin{array}{l}
z_{\mathrm{m}}=z_{0}-y_{0} \\
x_{\mathrm{m}}=0
\end{array}\right.
$$

Let us now consider a second cone (Fig. 8), located in $x_{1}, y_{1}$, and $z_{1}$ high. The equation of its intersection with the plane $y=0$ is

$$
\left(x-x_{1}\right)^{2}+y_{1}^{2}=\left(z-z_{1}\right)^{2}
$$

and its height in $x=0$, that is at the maximum of the first intersection, is

where

$$
\begin{aligned}
z(0) & =z_{1}-r_{c}, \\
r_{\mathrm{c}} & =\sqrt{x_{1}^{2}+y_{1}^{2}}
\end{aligned}
$$

That second intersection will overlap the maximum of the first one when

with

$$
z_{1}-r_{\mathrm{c}}>z_{0}-y_{0}
$$

$$
z_{1}-r_{\mathrm{c}}>z_{\mathrm{m}}
$$



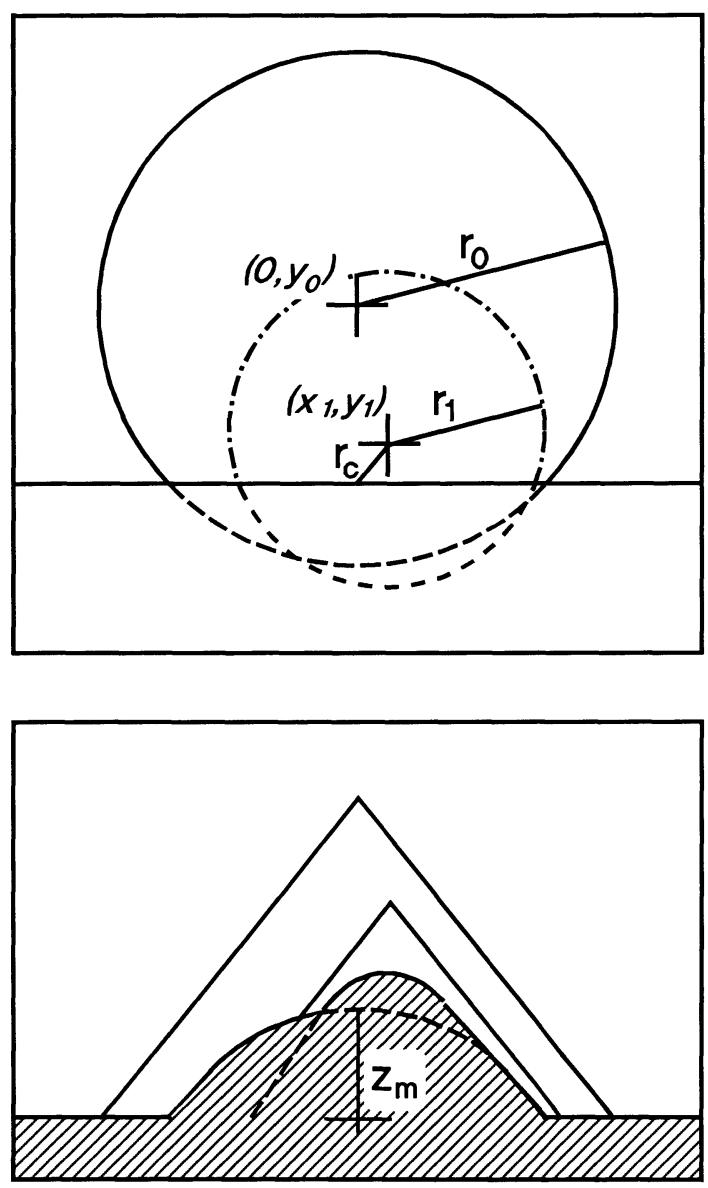

Fig. 8. - Possible overlapping of cones: a) base; b) cross sectional view. The hidden maximum will not be counted.

that is

$$
0<r_{\mathrm{c}}<z_{1}-z_{\mathrm{m}}
$$

That condition holds within a circular area as large as $\pi\left(z_{1}-z_{\mathrm{m}}\right)^{2}$, where the number of cones whose height lies between $z_{1}$ and $z_{1}+\mathrm{d} z_{1}$ is

$$
\sigma \pi\left(z_{1}-z_{\mathrm{m}}\right)^{2} f\left(z_{1}\right) \frac{\mathrm{d} z_{1}}{z_{\mathrm{M}}}
$$

of course, not all the values of $z_{1}$ give rise to an overlap: $z_{1}>z_{\mathrm{m}}$ is required in order to make it possible.

It follows that the mean number of cones able to hide the given section is given by:

$$
\frac{\sigma \pi}{z_{\mathrm{M}}} \int_{z_{\mathrm{m}}}^{z_{\mathrm{M}}}\left(z_{1}-z_{\mathrm{m}}\right)^{2} f\left(z_{1}\right) \mathrm{d} z_{1}
$$


The quantity above is a number, and should be regarded as the probability that the given section of the first cone is completely covered by any other cone. That number, indeed, could easily exceed unity, which merely means that the most probable situation is that more than one cone will cover the section. In order to deal with a correct expression for a probability, the distribution $p\left(z_{\mathrm{m}}\right)$ needs to be introduced:

$$
p\left(z_{\mathrm{m}}\right)=\operatorname{Inf}\left\{1 ; \frac{\pi \sigma}{z_{\mathrm{M}}} \int_{z_{\mathrm{m}}}^{z_{\mathrm{M}}}\left(q-z_{\mathrm{m}}\right)^{2} f(q) \mathrm{d} q\right\}
$$

It is possible now to correct the expression (2) by subtracting, where necessary, the contribution of the hidden sections. A new function $g\left(z_{\mathrm{m}}\right)$ can be defined now as the linear density of measured heights in the cross sectional plane:

$$
g\left(z_{\mathrm{m}}\right)=\frac{\left[1-p\left(z_{\mathrm{m}}\right)\right] \int_{z_{\mathrm{m}}}^{z} \mathrm{M} f(q) \mathrm{d} q}{\int_{0}^{z} \mathrm{M}\left[1-p\left(z_{\mathrm{m}}\right)\right] \int_{z_{\mathrm{m}}}^{z} \mathrm{M} f(q) \mathrm{d} q \mathrm{~d} z_{\mathbf{m}}}
$$

which is the final result.

The simple proportionality between this function and the distribution $f(z)$ is destroyed. Nevertheless, because of the greater significance of the higher tips in tunneling effects, it is important to observe that as $z_{\mathrm{m}}$ approaches $z_{\mathrm{M}}$ the value of $p\left(z_{\mathrm{m}}\right)$ vanishes. Moreover, when insulated tips are experimentally encountered, as in the reported pictures, an overall low density can be inferred, further reducing the value of $p\left(z_{\mathrm{m}}\right)$. This means that the relation (2) could be taken into account as the solution of the problem for the upper extreme of the height distribution, which is the most relevant part of the observation.

The same considerations lead to neglect at a first order the perturbative effect of the finite TEM specimen thickness: It could affect, indecd, just the lower part of the height distribution, corresponding to the smallest tips, which have no influence in tunneling and, in any case, are likely to be hidden by larger asperities in the experimental pictures.

3.2 AN EXAMPLE. - As a simple example, let be $f\left(z_{0}\right)$ an uniform distribution along the normalized height domain, that is:

$$
f\left(z_{0}\right)=1 \quad 0<z_{0}<1=z_{M}
$$

It follows:

$$
p\left(z_{\mathrm{m}}\right)=\operatorname{Inf}\left\{1 ; \frac{\pi \sigma}{3}\left(1-z_{\mathrm{m}}\right)^{3}\right\}
$$

that means:

$$
p\left(z_{\mathrm{m}}\right)= \begin{cases}1 & \text { when } z_{\mathrm{m}}<1-\left(\frac{3}{\pi \sigma}\right)^{1 / 3} \\ \frac{\pi \sigma}{3}\left(1-z_{\mathrm{m}}\right)^{3} & \text { when } z_{\mathrm{m}}>1-\left(\frac{3}{\pi \sigma}\right)^{1 / 3}\end{cases}
$$

The first condition, setting $p\left(z_{\mathrm{m}}\right)=1$, could be satisfied for some value of $z_{\mathrm{m}}$ only if $\sigma>3 / \pi$. Taking into account that the area of the base of the highest cone is $\pi$, this means that more than three cones are required to be centered on such an area in order to give that "total overlap" situation. 
It follows:

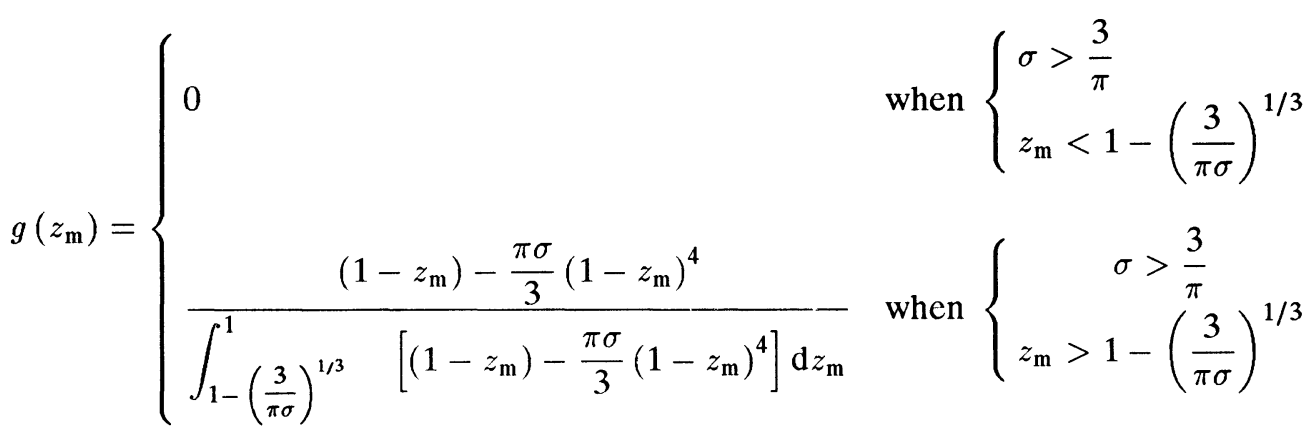

$$
\begin{aligned}
& g\left(z_{\mathrm{m}}\right)=\frac{\left(1-z_{\mathrm{m}}\right)-\frac{\pi \sigma}{3}\left(1-z_{\mathrm{m}}\right)^{4}}{\int_{0}^{1}\left[\left(1-z_{\mathrm{m}}\right)-\frac{\pi \sigma}{3}\left(1-z_{\mathrm{m}}\right)^{4}\right] \mathrm{d} z_{\mathrm{m}}}, \text { when } \sigma<\frac{3}{\pi}
\end{aligned}
$$

Indicating now as $C_{\sigma}$ the coefficient

$$
C_{\sigma}=\sup \left\{0 ; 1-\left(\frac{3}{\pi \sigma}\right)^{1 / 3}\right\}
$$

we obtain:

$$
g\left(z_{\mathrm{m}}\right)= \begin{cases}0 & , z_{\mathrm{m}}<C_{\sigma} \\ \alpha_{\sigma}\left[\left(1-z_{\mathrm{m}}\right)-\frac{\pi \sigma}{3}\left(1-z_{\mathrm{m}}\right)^{4}\right] & , z_{\mathrm{m}}>C_{\sigma}\end{cases}
$$

with

$$
\alpha_{\sigma}=\int_{C_{\sigma}}^{1}\left[\left(1-z_{\mathrm{m}}\right)-\frac{\pi \sigma}{3}\left(1-z_{\mathrm{m}}\right)^{4}\right] \mathrm{d} z_{\mathrm{m}}
$$

Figure 9 illustrates the behavior of $g$ according to two different values of the surface density of cones $\sigma$.

\section{Discussion.}

Two assumptions should be discussed in the above model: The conical shape of the tips and the location of the zero height plane, that means the problem of the absolute heights.

4.1 THE CONICAL SHAPE. - The conical shape of constant aperture is of course a simplification: The steam oxidation of a polycrystalline structure will produce asymmetries on the sides of the tips, smoothing of the vertex and some dispersion in the inclinations of the side walls.

Anyway, the orientation of the tips around their axis is likely to be casual, and in any case, the tunneling current has to be integrated on the receiving surface, that is over all the directions normal to the tip axis. A circular symmetry of the theoretical tip merely describes that statistical situation. 


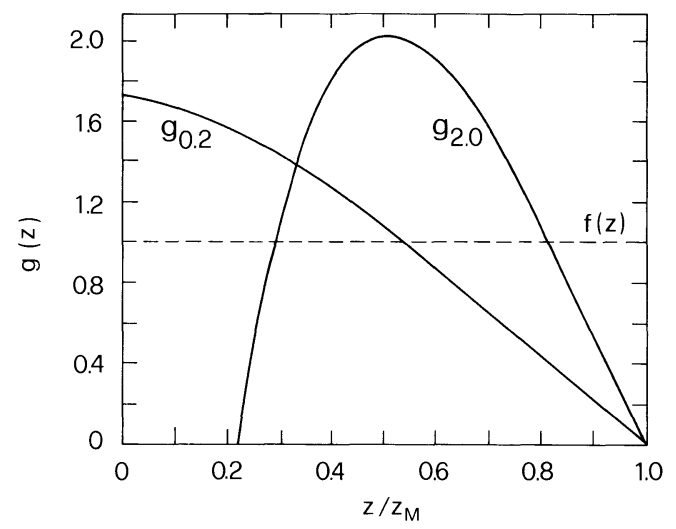

Fig. 9. - Distribution $g$ of the observed maxima in cross sectional view, generated by an uniform distribution $f$ of cone heights. The linear trend of $g$ for large values of $z$ shows the validity range of the relationship $f(z) g^{\prime}(z)$.

The fixed aperture of the cones, on the other hand can be thought once again as an average value over the actual distribution. That approach has the great advantage of describing the cones as a single parameter family, depending only on their height. A little dispersion, indeed, was observed on the TEM images, and the same smoothing of the edges, which complicates the simulation of the vertex, helps in reducing the dispersion over the apertures. In any case, the actual shape of the tips far from their vertex is likely to be unessential from the tunneling point of view.

The conical shape becomes critical at the vertex of the tip: The curvature of the equipotential surfaces, and consequently the tunneling transmission coefficient, is strictly related to the curvature of the emitting surface. A rounded tip will transform a cone in something like an hyperboloid, whose vertex would supply a lower tunneling current density on a broader area than a conical tip. Nevertheless, the above model could be modified in order to be suitably applied to that more realistic situation: as an example, the hyperbolic shape could be taken into account, resulting in a slight modification of the formulas (see appendix). The general behavior still holds, and the two models approach as the radius of curvature of the rounded tip becomes small.

4.2 Location of THE ZERo height Plane. - It is clear that any finite density of semiinfinite cones will identify a critical distance below the tips where complete overlap occurs. Beyond that distance the space is completely filled by one or more cones, and any possible vertex occuring in that region is hidden. Of course, an hidden distribution is equivalent to a null distribution, and this suggests that the problem of the location of the zero height plane is not a true problem: Many different choices could be made as well, all producing hidden distributions below a certain height limit. It follows that the zero height plane can be arbitrary. The real problem is to identify that value of $z$ beyond which overlap can be thought to be negligible, that means the region where the direct proportionality between $g(z)$ and $f^{\prime}(z)$ holds. Just as an empirical suggestion, supported by the expected low significance of the lower tips in tunneling processes, that region could be considered where $g(z)$ decreases with increasing $z$. As a last consideration, the deviation of the upper $\mathrm{SiO}_{2}$ /poly silicon interface from flatness (see Fig. 4 and Fig. 7a) should be taken into account. A mathematical treatment could be proposed, following the way traced the tips. Anyway, the corrospondence between the modulations of the interface and the underlying tips suggests to develope a common model for both $\mathrm{SiO}_{2}$ /poly silicon interfaces near a single tip. Their repre- 
sentation as two surfaces of a same family of rotational prolate hyperboloids, indeed, leads to a simple solution of the potential problem and allows a 3D treatment of the tunneling mechanism, as it will be reported in a next paper.

\section{Conclusion.}

A model is proposed to reconstruct a 3D distribution of microscopical conical tips on a flat surface starting from experimental observations of $2 \mathrm{D}$ cross sectional views of the same distribution. The results could be suitably applied to smooth conical shapes.

The approximations involved in the model look well justified in studying tunneling mechanisms from that distribution of tips.

The development of a 3D model for tunneling injection from a single tip should make the above analysis relevant for complete theoretical analysis of behaviour and degradation of structures as EEPROM cells under standard operating conditions.

\section{Acknowledgements.}

The authors wish to thank the staff of the electron microscopy group of CNR-LAMEL, Bologna, for the skillful assistance in specimen preparation and TEM observation. We are also indebted to Prof. G. Majni and Prof. C. Morandi for many suggestions and indications.

\section{Appendix.}

Let any tip be described by a function $h$ :

$$
h\left(z_{0}, x, y, x_{0}, y_{0}\right)=z_{0}-\Psi\left(\sqrt{\left(x-x_{0}\right)^{2}+\left(y-y_{0}\right)^{2}}\right)
$$

where $x, y$ is the intersection of the axis of the tip with the $z=0$ plane, and $\Psi(u)$ is a radial function which vanishes at $u=0$ (in the conical model one has $\Psi(u)=u$ ).

Following the previous way, let a tip be centered in $0, y$; the plane $y_{0}=0$ will intersect it along a curve whose maximum $z_{\mathrm{m}}$ is located at

$$
z_{\mathrm{m}}=h\left(z_{0}, 0, y, 0,0\right)=z_{0}-\Psi(y)
$$

The expression of $\mathrm{d} n\left(y, z_{\mathrm{m}}\right)$ is then modified as:

$$
\mathrm{d} n\left(z_{\mathrm{m}}\right)= \begin{cases}\sigma L f\left(\Psi(y)+z_{\mathrm{m}}\right) \mathrm{d} y \frac{\mathrm{d}\left(\Psi(y)+z_{\mathrm{m}}\right)}{z_{\mathrm{M}}} & \sim L f\left(\Psi(y)+z_{\mathrm{m}}\right) \mathrm{d} y \mathrm{~d} z_{\mathrm{m}} \\
& \text { when }\left\{\begin{array}{l}
0<z_{\mathrm{m}}<z_{\mathrm{M}} \\
\Psi(y)+z_{\mathrm{m}}<z_{\mathrm{M}}
\end{array}\right. \\
0 & \text { otherwise }\end{cases}
$$

where the square differential $\mathrm{d} y^{2} \cdot \frac{\mathrm{d} \Psi}{\mathrm{d} y}$ was indicated as negligible. 
The distribution $\mathrm{d} n\left(z_{\mathrm{m}}\right)$ is obtained again as a sum in $\mathrm{d} y$ of the above expression, which gives:

$$
\mathrm{d} n\left(z_{\mathrm{m}}\right)=\frac{\mathrm{d} z_{\mathrm{m}}}{z_{\mathrm{M}}} 2 \sigma L \int_{z_{\mathbf{m}}}^{z_{\mathrm{M}}} f(p) \Omega\left(p-z_{\mathrm{m}}\right) \mathrm{d} p
$$

where

$$
\Omega\left(p-z_{\mathrm{m}}\right)=R_{\mathrm{e}}\left[\frac{\mathrm{d}}{\mathrm{d} p} \Psi^{-1}\left(p-z_{\mathrm{m}}\right)\right]
$$

and is unity for any $y$ in the conical case.

The normalized distribution $\gamma\left(z_{\mathrm{m}}\right)$ is now:

$$
\gamma\left(z_{\mathrm{m}}\right)=\frac{\int_{z_{\mathrm{m}}}^{z_{\mathrm{M}}} f(p) \Omega\left(p-z_{\mathrm{m}}\right) \mathrm{d} p}{\int_{0}^{z} \mathrm{~d} q \int_{q}^{z} \mathrm{M} f(p) \Omega(p-q) \mathrm{d} p}
$$

In a very similar way, the overlap condition can be taken into account, leading to the expression of $p\left(z_{\mathrm{m}}\right)$ as:

$$
p\left(z_{\mathrm{m}}\right)=\operatorname{Inf}\left\{1 ; \frac{\pi \sigma}{z_{\mathrm{M}}} \int_{z_{\mathrm{m}}}^{z_{\mathrm{M}}}\left[\Psi^{-1}\left(q-z_{\mathrm{m}}\right)\right]^{2} f(q) \mathrm{d} q\right\}
$$

Integrals in both $\gamma\left(z_{\mathrm{m}}\right)$ and $p\left(z_{\mathrm{m}}\right)$ must be taken for non negative values of the argument. Negative values could arise from the evaluation of the formal inversion of the function $\Psi$ for values of $z_{\mathrm{m}}$ lying between the actual rounded apex and the vertex of the asymptotic cone.

In order to deal with a suitable example, let us consider any tip as a given hyperboloid with the apex located at a given height:

$$
\begin{gathered}
\Psi(r)=\sqrt{a^{2}+r^{2}} \\
h\left(z_{0}, x, y, x_{0}, y_{0}\right)=z_{0}-\sqrt{a^{2}+\left(x-x_{0}\right)^{2}+\left(y-y_{0}\right)^{2}}
\end{gathered}
$$

with the indicated choice of the parameters, the hyperboloid has the cone $z_{0}-\sqrt{\left(x-x_{0}\right)^{2}+\left(y-y_{0}\right)^{2}}$ as its asymptotic surface. The following expressions hold for the relevant formulas:

$$
\begin{gathered}
z_{\mathrm{m}}=z_{0}-\sqrt{a^{2}+y^{2}} \\
\mathrm{~d} n\left(z_{\mathrm{m}}\right)= \begin{cases}\sigma L f\left(z_{\mathrm{m}}+\sqrt{a^{2}+y^{2}}\right) \mathrm{d} y \mathrm{~d} z_{\mathrm{m}} & , 0<z_{\mathrm{m}}<z_{\mathrm{M}} \\
0 & , \text { otherwise }\end{cases} \\
\Omega\left(p-z_{\mathrm{m}}\right)= \begin{cases}\frac{p-z_{\mathrm{m}}}{\sqrt{\left(p-z_{\mathrm{m}}\right)^{2}-a^{2}}}, & \left(p-z_{\mathrm{m}}\right)^{2}>a^{2} \\
0 \quad, \text { otherwise }\end{cases} \\
\mathrm{d} n\left(z_{\mathrm{m}}\right)=\frac{\mathrm{d} z_{\mathrm{m}}}{z_{\mathrm{M}}} 2 \sigma L \int_{z_{\mathrm{m}}+a}^{z} \frac{f(p)\left(p-z_{\mathrm{m}}\right)}{\sqrt{\left(p-z_{\mathrm{m}}\right)^{2}-a^{2}}} \mathrm{~d} p
\end{gathered}
$$




$$
\begin{gathered}
\gamma\left(z_{\mathrm{m}}\right)=\frac{\int_{z_{\mathrm{m}}+a}^{z_{\mathrm{M}}} f(p) \frac{p-z_{\mathrm{m}}}{\sqrt{\left(p-z_{\mathrm{m}}\right)^{2}-a^{2}}} \mathrm{~d} p}{\int_{0}^{z_{\mathrm{M}}^{-a}} \mathrm{~d} q \int_{q+a}^{z \mathrm{M}} f(p) \frac{p-q}{\sqrt{(p-q)^{2}-a^{2}}} \mathrm{~d} p} \\
p\left(z_{\mathrm{m}}\right)=\operatorname{Inf}\left\{1 ; \frac{\pi \sigma}{z_{\mathrm{M}}} \int_{z_{\mathrm{m}}}^{z_{\mathrm{M}}}\left[\left(q-z_{\mathrm{m}}\right)^{2}-a^{2}\right] f(q) \mathrm{d} q\right\}
\end{gathered}
$$

When the same constant distribution $f(z)=1, z_{\mathrm{M}}=1$, is considered, a straightforward calculation leads to $g\left(z_{\mathrm{m}}\right)$ :

$$
g\left(z_{\mathrm{m}}\right)=\frac{\left[1-p\left(z_{\mathrm{m}}\right)\right] \sqrt{\left(1-z_{\mathrm{m}}\right)^{2}-a^{2}}}{\int_{0}^{1-a}\left[1-p\left(z_{\mathrm{m}}\right)\right] \sqrt{\left(1-z_{\mathrm{m}}\right)^{2}-a^{2}} \mathrm{~d} z_{\mathrm{m}}}
$$

where

$$
p\left(z_{\mathrm{m}}\right)=\operatorname{Inf}\left\{1 ; \frac{\pi \sigma}{3}\left[\left(1-z_{\mathrm{m}}\right)^{3}+a^{2}\left(2 a-3-3 z_{\mathrm{m}}\right)\right]\right\}
$$

It is evident that when the parameter $a$ vanishes, this result is the same as in the conical case.

\section{References}

[1] LENZLinger M. and SNOw E.H., Fowler-Nordheim tunneling into thermally grown $\mathrm{SiO}_{2}$, J. Appl. Phys. 40 (1969) 278.

[2] KRIEGER G. and SwANSON R.M., Fowler-Nordheim electron tunneling in thin $\mathrm{Si}_{-} \mathrm{SiO}_{2}$-Al structures, J. Appl. Phys. 52 (1981) 5710.

[3] ELLIS R.K., Fowler-Nordheim emission from non planar surfaces, IEEE Trans. Electron Device Lett. EDL-3 (1982) 330.

[4] ELLIS R.K., WEGENER H.A.R. and CAYWOOD J.M., Electron tunneling in non-planar floating gate memory structure, IEEE Trans. IEDM 82 (1982) 749.

[5] HARARY E., Conduction and trapping of electrons in highly stressed ultrathin films of thermal $\mathrm{SiO}_{2}$, Appl. Phys. Lett. 30 (1977) 601.

[6] EIMANN P.A., MURARKA S.P. and SHENG T.T, Electrical conduction and breakdown in oxides of polycristalline silicon and their correlation with interface texture, J. Appl. Phys. 53 (1982) 6240.

[7] PRICKETT B.L., CAYWOOD J.M. and ElLIS R.K., Trapping in tunnel oxides grown on textured polysilicon, IEEE Trans. IRPS (1983) 114.

[8] HORIGUCHI S., KOBAYASHI T. and SAITO K., Interface-trap generation modelling of Fowler-Nordheim tunnel injection into ultra-thin gate, J. Appl. Phys. 58 (1985) 387.

[9] KLEIN N. and NEVANLINNA O., Lowering of the breakdown voltage of silicon dioxide by asperities and at spherical electrodes, Solid-State Electron. 26 (1983) 883.

[10] WEGENER H.A.R., Endurance of XICOR EEPROM and NOVRAMs, Data Book Xicor (1988).

[11] KAHNG D. and SZE S.M., Floating gate and its applications on memory device, Bell Syst. Techn. J. 46 (1967) 1283.

[12] KOLODNY A., NIEH S.T.K., EITAN B. and SHAPPIR J., Analysis and modeling of floating gate EEPROM cells, IEEE Trans. Electron Devices 33, (1986) 835.

[13] Leyds J.L., Dufeutrelle O., Le Bihan R., Karray F. and Plantard M., Polysilicon oxidation studies: application to double polysilicon devices - Insulating films on semiconductors, Eds. J.J. Simonne and J. Buxo (Elsevier Science Publishers B.V., North-Holland) 1986. 
[14] FARAONE L., Properties of textured oxide/polysilicon interfaces - Insulating films on semiconductors, Eds. J.J. Simonne and J. Buxo (Elsevier Science Publishers B.V., North-Holland) 1986.

[15] KoRMA E.S., PEEK H.L. and VERWEY J.F., The influence of poly-silicon surface texturing on the $\mathrm{SiO}_{2}$ conduction and breakdown phenomena - Insulating films on semiconductors, Eds. J.J. Simonne and J. Buxo (Elsevier Science Publishers B.V., North-Holland), 1986.

[16] MAES H.E., WITTERS J. and GROESENEKEN G., Trends in non-volatile memory devices and technologies ESSDERC87 (1987) 743.

[17] BISSCHOP J., KORMA E.J., BOTTA E.F.F. and VERWEY J.F., A model for electrical conduction in polysilicon oxide, IEEE Trans. Electron. Devices 33 (1986) 1089.

[18] BissCHOP J., KORMA E.J., BOTTA E.F.F., SNIJDER J. and VERWEY J.F., Enhanced electron injection from the poly-Si $/ \mathrm{SiO}_{2}$ interface - Insulating films on semiconductors, Eds. J.J. Simonne and J. Buxo (Elsevier Science Publishers B.V., North-Holland), 1986.

[19] ROY A., LIBISCH F.R. and WHITE M.H., Electron tunneling from polysilicon asperities into poly-oxydes, solid-state Electronics 32, (1989) 655.

[20] Garulli A., ARMigliato A. and VANZi M., Preparation of cross-sections of silicon specimens for transmission electron microscopy, J. Microsc. Spectrosc. Electron. 10 (1985) 135.

Cet article a été imprimé avec le Macro Package "Editions de Physique Avril 1990". 